\title{
Biased ligand bonds to make right calls-laboratory of signal transduction reveals molecular mechanism for functional selectivity of GPCR
}

\author{
WANG Jue \\ Institute of Molecular Medicine, Peking University, Beijing 100871, China
}

Received May 21, 2014; accepted June 6, 2014; published online June 29, 2014

Citation: Wang J. Biased ligand bonds to make right calls—laboratory of signal transduction reveals molecular mechanism for functional selectivity of GPCR Sci China Life Sci, 2014, 57: 957-958, doi: 10.1007/s11427-014-4695-1

In a recent study, Professor Rui-Ping Xiao and her research group at Institute of Molecular Medicine of Peking University has provided an essential experimental evidence to support the new paradigm of functional selectivity for $G$ protein-coupled receptor (GPCR) signal transduction. The study was published online on May 15 in The Journal of Biological Chemistry [1].

The GPCR superfamily, a class of membrane proteins responsible for transmembrane signal transduction, has been a focal point in the field of biomedical research as well as drug discovery and development. Drugs targeting this superfamily account for about $50 \%$ of all prescription pharmaceuticals on the market. Recently, a paradigm called functional selectivity of GPCR signaling has been proposed to explain how different ligands can cause a single GPCR to relay diverse downstream signals. According to this paradigm, ligand-specific receptor signaling (biased signaling) depends on ligand-specific receptor conformation. However, this understanding suffers from the lack of structure-function-based experimental evidence.

As early as 2003 [2], Rui-Ping Xiao and her team discovered that while most $\beta_{2}$-adrenergic receptor $\left(\beta_{2}-\mathrm{AR}\right)$ agonists stimulate the receptor to activate both the stimulatory $G$ proteins $\left(G_{s}\right)$ and the inhibitory $G$ proteins $\left(G_{i}\right)$ to produce dual signaling, fenoterol stimulates the $G_{\mathrm{s}}$-selective
$\beta_{2}$-AR signaling, but the molecular mechanism for the functional selectivity of $\beta_{2}$-AR signaling remains elusive.

The latest study by Woo et al. [1] demonstrated that "tyrosine 308 is necessary for $\mathrm{G}_{\mathrm{s}}$-biased signaling of $\beta_{2}$-AR". The researchers found that $\left(R, R^{\prime}\right)-4^{\prime}$-aminofenoterol, an analog of fenoterol, stimulates the wild-type $\beta_{2}$-AR to produce selectively $G_{s}$ signaling but stimulates the $\beta_{2}$-AR Y308F mutant to produce $G_{s}$ and $G_{i}$ dual signaling. Further application of a cohort of fenoterol derivatives in computer modeling studies and biological assays on cardiomyocytes led to the identification of a hydrogen bond interaction between the 4'-O or 4'-N of the ligand and the phenyl hydroxyl group of $\beta_{2}$-AR-Y308 necessary for $G_{\mathrm{s}}$-biased signaling (Figure 1).

The study reported for the first time the identification of a ligand-receptor interaction dedicated to functional selectivity. It also revealed the structural basis of functional selectivity for $\beta_{2}$-AR signaling and provided structural insights for structure-based design of signaling pathwayspecific drugs.

The study was funded by the National Basic Research Program of China, National Science Foundation of China, Beijing Key Laboratory of Cardiometabolic Molecular Medicine, Peking University, Intramural Research Program of the United States, National Institutes of Health, National Institute on Aging, and the Foundation of Polish Science.

*Corresponding author (email: jue.wang @ pku.edu.cn) 


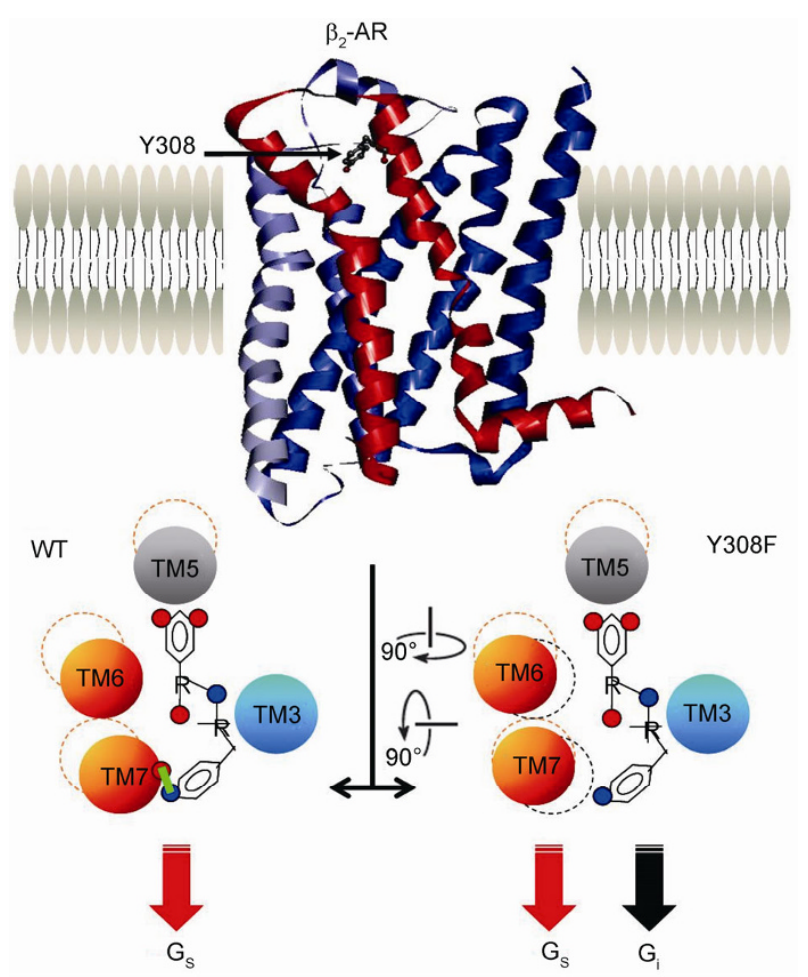

Figure 1 Schematic diagrams of different conformations and signalings in $\beta_{2}$-AR and $\beta_{2}$-AR Y308F mutant. Molecular model of $\beta_{2}$-AR (pdb:2RH1) embedded in lipid bilayer depicting Y308 on transmembrane helix (TM) 7 near the extracellular surface (top panel). Extracellular views of the wild-type (WT) $\beta_{2}$-AR (bottom left panel) and the $\beta_{2}$-AR Y308F mutant (bottom right panel). Only TMs 3, 5, 6 and 7 are shown since they form the ligand binding pockets of $\beta_{2}$-AR. $\left(R, R^{\prime}\right)-4^{\prime}$-Aminofenoterol (schematic structure with 2 Rs and 2 benzenes) is positioned in each of the ligand binding pockets of the WT and Y308F receptors based on molecular modeling data. Small red dots represent hydroxyl groups while small blue dots represent amino groups. Orange dotted circles represent the positions of TMs 5, 6 and 7 relative to TM 3 of the $\beta_{2}$-ARs in the inverse agonist-bound conformation. Solid circles represent the positions of the TMs in functionally selective conformations. The tentative positions of TMs 6 and 7 in functionally selective conformation of the WT $\beta_{2}$-AR are additionally shown as black dotted circles in the bottom right panel. Studies has shown that the movement of TM 6 at the cytoplasmic end is the greatest among the TMs during $\beta_{2}$-AR activation and has a direct effect on $\mathrm{G}_{\mathrm{s}}$ protein coupling although the movement at the ligand binding region is very subtle. A hydrogen bond interaction between the 4'-amino group of the ligand and the phenyl hydroxyl group of Y308 is depicted as a green line in the WT $\beta_{2}$-AR model (bottom left). This hydrogen bond interaction is absent in the corresponding $\beta_{2}$-AR Y308F mutant model (bottom right). It is postulated that the $\mathrm{G}_{\mathrm{s}}$-biased $\beta_{2}$-AR agonist $\left(R, R^{\prime}\right)-4^{\prime}$-aminofenoterol interacts specifically via a hydrogen bond with $\beta_{2}$-AR-Y308 and results in a receptor conformation that selectively activates $\mathrm{G}_{\mathrm{s}}$ signaling. In contrast, due to the absence of the key hydrogen bond, the $\mathrm{G}_{\mathrm{s}}$-baised agonist induces a different receptor conformation (particularly the change in TM 6 position) in the $\beta_{2}$-AR Y308F mutant. The result is the activation of promiscuous $\mathrm{G}_{\mathrm{s}}$ and $\mathrm{G}_{\mathrm{i}}$ dual signaling. The TMs of $\beta_{2}$-AR, ligand molecules and their positions are not drawn to scales. The functionally selective conformations shown in the diagram are not necessarily equivalent to active conformations. Rather, they should be more appropriately understood as intermediate states during ligand binding-induced conformational transition.

1 Woo AY, Jozwiak K, Toll L, Tanga MJ, Kozocas JA, Jimenez L, Huang Y, Song Y, Plazinska A, Pajak K, Paul RK, Bernier M, Wainer IW, Xiao RP. Tyrosine 308 is necessary for ligand-directed Gs-biased signaling of $\beta_{2}$-adrenoceptor. J Biol Chem, 2014, doi: 10.1074/jbc.M114.558882

2 Xiao RP, Zhang SJ, Chakir K, Avdonin P, Zhu W, Bond RA, Balke CW, Lakatta EG, Cheng H. Enhanced G(i) signaling selectively negates $\beta_{2}$-adrenergic receptor (AR) — but not $\beta_{1}$-AR-mediated positive inotropic effect in myocytes from failing rat hearts. Circulation, 2003, 108: 1633-1639

Open Access This article is distributed under the terms of the Creative Commons Attribution License which permits any use, distribution, and reproduction in any medium, provided the original author(s) and source are credited. 